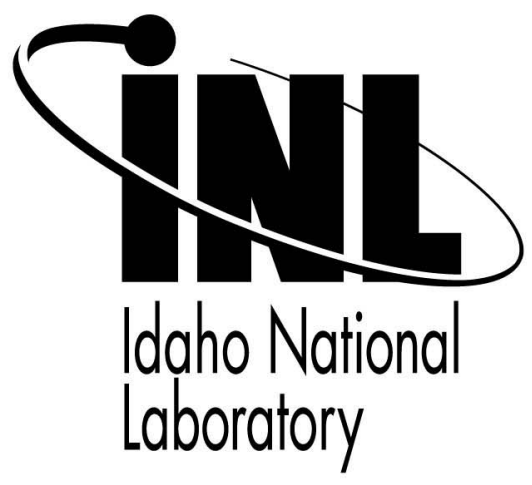

\title{
A Method for Selecting Software for Dynamic Event Analysis II: The Taylor Anvil and Dynamic Brazilian Tests
}

ICONE 16

J. M. Lacy

S. R. Novascone

W. D. Richins

T. K. Larson

May 2008

This is a preprint of a paper intended for publication in a journal or proceedings. Since changes may be made before publication, this preprint should not be cited or reproduced without permission of the author. This document was prepared as an account of work sponsored by an agency of the United States Government. Neither the United States Government nor any agency thereof, or any of their employees, makes any warranty, expressed or implied, or assumes any legal liability or responsibility for any third party's use, or the results of such use, of any information, apparatus, product or process disclosed in this report, or represents that its use by such third party would not infringe privately owned rights. The views expressed in this paper are not necessarily those of the United States Government or the sponsoring agency. 


\section{A METHOD FOR SELECTING SOFTWARE FOR DYNAMIC EVENT ANALYSIS II: THE TAYLOR ANVIL AND DYNAMIC BRAZILIAN TESTS}

\author{
J. M. Lacy \\ Applied Mechanics Department \\ W. D. Richins \\ Applied Mechanics Department
}

\author{
S. R. Novascone \\ Applied Mechanics Department
}

T. K. Larson

Infrastructure Systems Analysis Department

Idaho National Laboratory

Idaho Falls, Idaho 83415

\begin{abstract}
New nuclear power reactor designs will require resistance to a variety of possible malevolent attacks as well as traditional dynamic accident scenarios. The design/analysis team may be faced with a broad range of phenomena including air and ground blasts, high-velocity penetrators or shaped charges, and vehicle or aircraft impacts. With a host of software tools available to address these high-energy events, the analysis team must evaluate and select the software most appropriate for their particular set of problems. The accuracy of the selected software should then be validated with respect to the phenomena governing the interaction of the threat and structure.

Several software codes are available for the study of blast, impact, and other shock phenomena. At the Idaho National Laboratory (INL), a study is underway to investigate the comparative characteristics of a group of shock and high-strain rate physics codes including ABAQUS, LS-DYNA, CTH, ALEGRA, ALE3D, AUTODYN, and RADIOSS.

In part I of this report published in 2007, a series of five benchmark problems to exercise some important capabilities of the subject software was identified. The benchmark problems selected are a Taylor cylinder test, a split Hopkinson pressure bar test, a free air blast, the dynamic splitting tension (Brazilian) test, and projectile penetration of a concrete slab. Part II-- this paper-- reports the results of two of the benchmark problems: the Taylor cylinder and the dynamic Brazilian test.

The Taylor cylinder test is a method to determine the dynamic yield properties of materials. The test specimen is a right circular cylinder which is impacted against a theoretically rigid target. The cylinder deforms upon impact, with the final shape depending upon the specimen density, the impact
\end{abstract}

velocity, and the dynamic yield stress, in turn a function of strain and strain rate.

The splitting tension test, or Brazilian test, is a method to measure the tensile strength of concrete using a cylindrical specimen. The specimen is loaded diametrically in compression, producing a fracture at the center of the specimen that propagates toward the loading points until the cylinder is split. To generate a dynamic load, different methods such as a drop-weight or a split Hopkinson pressure bar are employed.

The Taylor anvil and dynamic Brazilian test analyses are presented, including discussion of the analysis approach for each of the five subject software packages and two vendor submittals; comparison of results both among the codes and to physical test results; and conclusions as to the applicability of the subject codes to these two problems. Studies of the remaining three benchmark problems and overall conclusions will be presented in future publications.

\section{INTRODUCTION}

As presented in Part I [1], we anticipate that the revived interest in nuclear power will lead to a growing need for economical design solutions to maintain the safety and integrity of new generation nuclear power plants with respect to severe dynamic loads, whether accidental or malevolent.

Aside from possible high-energy accident scenarios, which depend on reactor design parameters, potential attackers have a wide variety of tools which they may direct at a high-visibility, high-consequence target such as a nuclear power facility. The design basis threat may include attacks by vehicle, aircraft, high explosives, ballistic penetrators, explosively formed projectiles, and shaped charges. 
Design teams will likely employ solid dynamics/shock physics software (hydro codes) to simulate the effects of postulated accidents and attacks on various elements of their reactor plant system. The designers' first decision will be to choose the best simulation software for the particular problem at hand. A comparison among the different software products would appear to be a useful method to select the best tool.

This INL project is in the second year of a 3-year effort to develop and implement a comparative study of a broad group of solid dynamics and shock physics simulation codes. Our objectives are to develop a manageably small yet broadly representative benchmarking suite and apply it to a large fraction of the software available. We will report on usability, suitability to the class of problem, computational efficiency, and accuracy. In Part I, we discussed our method for selecting a benchmarking problem suite, the problems that make up the suite, and some preliminary results from a subset of codes. Part II presents the results for two of the benchmark problems: the Taylor cylinder test and the split-cylinder (Brazilian) test.

\section{SOFTWARE EMPLOYED}

The software packages selected for this study are

- ABAQUS [2]

- LS-DYNA [3]

- CTH [4]

- ALEGRA [5]

- ALE3D [6]

This code group is not intended to be exhaustive and simply represents software accessible to the authors for reasons related to software license availability and limits on funding and time. In addition to these codes available at INL, both benchmark problems described in this paper were solved using AUTODYN [7] and RADIOSS [8] by the respective vendors.

\section{BENCHMARK PROBLEM DESCRIPTIONS}

Our rationale for selecting 5 representative benchmark problems from the literally hundreds available is presented in Part 1. To re-state briefly, problems were selected to be; 1) relatively uncomplicated, so that they may be coded and run in a realistic quantity of time, 2) designed to engage a limited set of physical phenomena per problem, so that the source of any differences in results may be more easily identified, 3) independent of the code formulation for broad applicability, 4) verifiable, either by closed-form solution or experimental result, and 5) unlimited in distribution, so that results may be compared among institutions with different computing hardware resources.

In no particular order, the 5 problems selected for the study are

- Taylor cylinder test

- Free air blast

- Split Hopkinson pressure bar

- Dynamic split-cylinder test
- Projectile penetration of a concrete slab.

Attributes of each of the problems are presented in Part I. The remainder of this paper discusses our experiences and results applying our software group to the Taylor cylinder and dynamic split-cylinder problems.

\section{TAYLOR CYLINDER TEST}

\section{Description}

The Taylor cylinder test employs a right circular cylinder of the subject material which is impacted against a theoretically rigid target. The test was originally developed by Taylor [9]. Assuming that the impact surface was rigid, with perfectly plastic material behavior and 1-D wave propagation, the test could be used to determine the dynamic yield stress from measurements of impact velocity and comparison of the undeformed and deformed specimen length. Figure 1 shows a photo of a Taylor cylinder after impact.

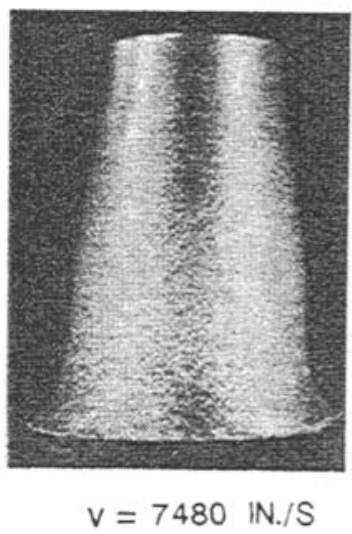

Figure 1 OFHC Taylor cylinder after impact at a velocity of $190 \mathrm{~m} / \mathrm{s}(7480 \mathrm{in} / \mathrm{s})$ from Johnson and Holmquist [10]

Currently, the Taylor cylinder test is used chiefly to validate constitutive models by direct comparison of the experimental final deformed cylinder shape to computational predictions. The final deformed shape depends upon the specimen density, impact velocity, dynamic yield stress, strain hardening, and adiabatic deformation heating, which in turn depend on strain and strain rate fields that vary both temporally and spatially in the specimen. Taylor impact simulations are also used to evaluate modeling features such as rigid and symmetric boundary conditions, plastic wave propagation, and large element distortion.

The benchmark Taylor cylinder problem used an oxygenfree, high conductivity (OFHC) copper rod $0.381 \mathrm{~cm}(0.15 \mathrm{in})$ diameter by $2.54 \mathrm{~cm}$ (1 in.) long striking a rigid boundary at velocities of 13000,14600 , and $19000 \mathrm{~cm} / \mathrm{s}(5120,5750$, and $7480 \mathrm{in} / \mathrm{sec}$, respectively) to match the experimental data reported by Johnson and Holmquist [10]. Reference [10] reports the specimen material had a Rockwell Hardness of F- 
30, average grain size $0.00762 \mathrm{~cm}$, density $8.96 \mathrm{gm} / \mathrm{cm}^{3}$, specific heat $3.83(10)^{6} \mathrm{~cm}^{2} /\left(\mathrm{C}-\mathrm{s}^{2}\right)$, and melting temperature $1082^{\circ} \mathrm{C}$.

The Johnson-Cook [11] constitutive model was employed for all simulations, with consistent parameters defined in Table 1. As discussed below, a bilinear elastic-plastic kinematic hardening model was also employed using one code (ALE3D) to investigate the sensitivity of the results to the material model. Equation of state (EOS) models were employed in each of the simulations, but due to the relatively low energies the EOS models are thought to be of secondary importance.

Table 1. Johnson-Cook Parameters for OFHC copper

\begin{tabular}{|l|l|l|}
\hline Parameter & CGS units & English Units \\
\hline A & $8.963 \mathrm{e}+8 \mathrm{dy} / \mathrm{cm}^{2}$ & $1.30 \mathrm{e}+4 \mathrm{psi}$ \\
\hline $\mathrm{B}$ & $2.9164 \mathrm{e}+9 \mathrm{dy} / \mathrm{cm}^{2}$ & $4.23 \mathrm{e}+4 \mathrm{psi}$ \\
\hline $\mathrm{n}$ & 0.31 & 0.31 \\
\hline $\mathrm{m}$ & 1.09 & 1.09 \\
\hline $\mathrm{C}$ & 0.025 & 0.025 \\
\hline Tmelt & $1356 \mathrm{~K}$ & $2441^{\circ} \mathrm{R}$ \\
\hline Specific Heat & $3.83 \mathrm{e}+6 \mathrm{erg} / \mathrm{g}-\mathrm{K}$ & $9.15 \mathrm{e}-2 \mathrm{BTU} / \mathrm{lb}-{ }^{\circ} \mathrm{F}$ \\
\hline
\end{tabular}

ABAQUS - For the ABAQUS simulation, a full 3-dimensional model was developed. The model consisted of 104000 hexahedral elements and 109200 nodes, with an average element dimension of $0.03 \mathrm{~cm}$. The Johnson-Cook plasticity model was called with the parameter values stated in Table 1 . The cylinder velocity was set with an initial velocity condition, and the anvil was represented with a no-displacement condition on the forward-end nodes in the axial direction. The Explicit solver was used. The displaced shape profile was created with a node output call on a nodeset defined at the intersection of the cylinder surface and the ZX plane. The entire process was very straightforward with no difficulties encountered.

LS-DYNA - The LS-DYNA mesh and boundary conditions were identical to the ABAQUS model using Contact_Automatic_Single_Surface with the soft constraint option set to 2, and a rigid planar surface used as the target. The material model was Material 15, Johnson_Cook.

CTH - The CTH model was 2D with a mesh size of $0.02 \mathrm{~cm}$ on a side. The Johnson-Cook model with the properties described in Table 1 was employed. A velocity was prescribed to the material in the Eulerian mesh representing the copper with a rigid and frictionless boundary condition at the end of the cylinder. The final deformed shaped was saved in JPEG format and measured with a plot digitizer. No unusual difficulties were encountered.

ALEGRA- The Alegra model was 2D with a mesh size of 0.01 $\mathrm{cm}$ on a side. The Johnson-Cook model with the properties described in Table 1 was employed. A velocity was prescribed to the material in the Eulerian mesh representing the copper with a rigid and frictionless boundary condition at the end of the cylinder. The final deformed shaped was saved in JPEG format and measured with a plot digitizer. No unusual difficulties were encountered.

ALE3D - Two different geometry models were employed for the ALE3D investigation of the Taylor anvil problem including a 2-D r-z rectangular geometry consisting of 320 mesh elements, 369 nodes, one domain, and one region (the copper) and a 3-D 1/4-cylinder geometry composed of 1920 mesh elements, 2501 nodes, one domain, and one region (copper). The Johnson-Cook model with the properties described in Table 1 was employed. Additionally, a bilinear elastic-plastic kinematic hardening model was employed. The material constants used for this model were, density $8.93 \mathrm{gm} / \mathrm{cm}^{3}$, Young's elastic modulus $1.17(10)^{12} \mathrm{dy} / \mathrm{cm}^{2}$, yield point $8.99(10)^{8} \mathrm{dy} / \mathrm{cm}^{2}$, tangent modulus of $6.90(10)^{9} \mathrm{dy} / \mathrm{cm}^{2}$, and a Poisson's ratio of 0.33 . An initial velocity was assigned to all the nodes in the cylinder. A rigid and frictionless boundary condition was applied at one end of the cylinder. As an example of the models used in this analysis, an image of the ALE3D model is shown in Figure 2. There was no significant difference between the $2 \mathrm{D}$ and $3 \mathrm{D}$ results. The final deformed shape was saved as a graphics file and the profile digitized for comparison to experimental data. No difficulties were encountered running the computation.

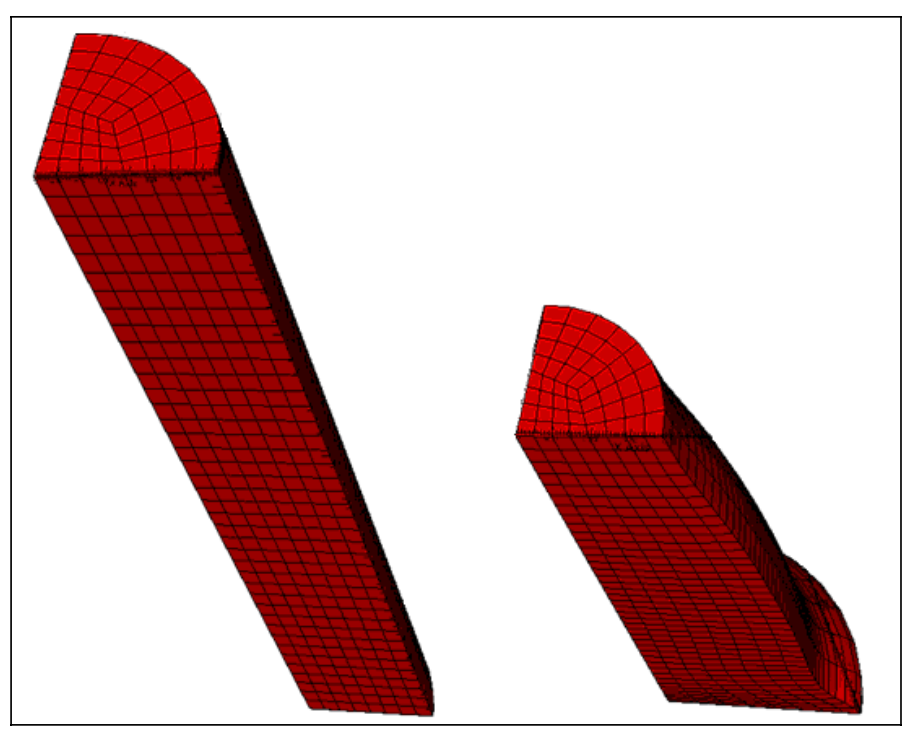

Figure 2. Example quarter-symmetry finite-element model

\section{Discussion}

Implementation of this problem was straightforward in all the codes evaluated. All the codes include a plethora of yield models for ductile metals, including the desired Johnson-Cook. The geometry is simple enough to create a mesh in minutes, whether using external pre-processors or the in-line mesh generation features of some of the codes $(\mathrm{CTH}$, Alegra, ALE3D). The non-deforming boundary against which the cylinder was impacted was trivial to implement in all cases. At the velocities studied, deformation of the rod did not cause undue distortion of Lagrangian elements, making the solution 
problem-free in all cases. Thus, from the user perspective we did not see any advantage of one code over another that could not be attributed to user inexperience.

Results of the various simulations are compared to experimental data reported by Johnson and Holmquist [10] in Figure 3. Due to space considerations, only the data for impact velocity of $19000 \mathrm{~cm} / \mathrm{s}$ is reported here, but results are similar at 13000 and $14600 \mathrm{~cm} / \mathrm{sec}$ velocities.

As Figure 3 illustrates, all results employing the reference Johnson-Cook material model were very nearly identical, regardless of formulation, mesh size, or dimensionality (2-, 2.5-, and 3-dimensional). While the different code results lie nearly on top of one another, they did not accurately match the reference experimental data. Deformation at the toe of the cylinder is over-predicted, while radial bulging near the center of the specimen and reduction of length are under-predicted. To investigate, we conducted a simple study to evaluate other constitutive relations in one code-ALE3D. One of those results, employing the bilinear elastic-plastic yield model distributed with the code (mentioned above) is shown as the trace of gray dots in Figure 3. This model very nearly matched the length reduction and central bulge features, while underpredicting the toe deformation. This result highlights the value of the Taylor cylinder test in its sensitivity to the material constitutive model employed.

One reason for the observed sensitivity is the broad range of strains and strain rates imposed through the body of the cylinder specimen. In the case of the $19000 \mathrm{~cm} / \mathrm{sec}$ impact, the central base area of the cylinder attains a plastic strain magnitude over 1.3, at a strain rate of approximately $20,000 \mathrm{~s}^{-1}$. At the same time, the top of the cylinder only sees strains of less than $1 \%$, with a corresponding rate on the order of $100 \mathrm{~s}^{-1}$. In order to successfully match the deformed specimen shape, the material model employed must accurately reflect the material's response over 4 orders of magnitude strain rate, to strains well over $100 \%$.

While the Taylor cylinder test is an excellent tool for evaluating numerical material models, we found it less useful as a benchmarking problem. The results from the different codes, and the level of effort required to achieve those results were very comparable, with very little to differentiate one from another. On the other hand, we would strongly recommend it as a tutorial problem for analysts new to a particular software package. The Taylor cylinder test could also be used to validate shock and high-strain rate physics codes not exercised in this effort.

In order to more strongly distinguish the capabilities of the subject codes, experimental data at higher impact velocities could be simulated, wherein large deformations necessitate the capabilities of Eulerian, ALE, and SPH formulations, and the formation and growth of radial cracks in the specimen ("petalling") further challenge the code capabilities.
Taylor Rod $19000 \mathrm{~cm} / \mathrm{s}$

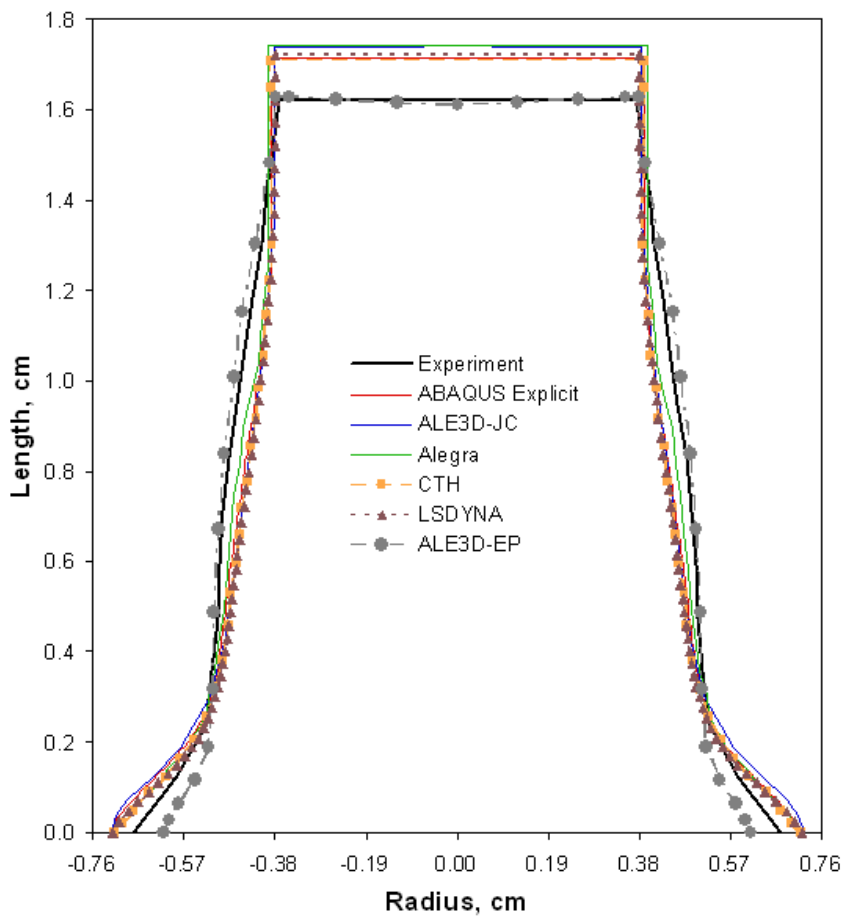

Figure 3. Taylor cylinder simulation and experimental data, $19000 \mathrm{~cm} / \mathrm{sec}$

\section{DYNAMIC SPLIT-CYLINDER (BRAZILIAN) TEST}

\section{Description}

Dynamic analysis software employed for nuclear reactor plant applications must have the ability to simulate the dynamic failure of concrete as well as its elastic properties. The splitcylinder test (also known as the Brazilian or splitting-tensile test) was chosen as a benchmark problem to address the ability of the software to predict the response of concrete to impact loading. The split-cylinder test subjects a concrete cylinder to diametric compression, inducing a nearly uniform tensile stress field perpendicular to the line of loading. ASTM standard C 496/C 496M-04 [12] defines the test at quasi-static load rates and a useful quantity of discussion in the literature addresses dynamic tests both experimentally and numerically.

The split-cylinder test differs from direct tension and compression tests in that the specimen is loaded diametrically in compression (Figure 4), rather than axially. A concrete cylinder is compressed on its diameter between two spacers (bearing strips between the cylinder and platens) of width $1 / 12$ the diameter of the cylinder. To generate a high-rate load, a split Hopkinson pressure bar (SHPB) is often employed using a smaller concrete test sample. Hughes et al [13] established that stress distribution in dynamic tests follows the classic elastic description. 
The stress field produces a fracture at the center of the specimen that propagates toward the loading points until the cylinder is split. Small zones of crushing failure are also observed near the supports [14]. This fracture and crushing response is difficult to address with classical finite element methods, and most investigators develop their own material models, finite elements, or both. For the purposes of this study, we employed elements and material models distributed with the software to investigate their off-the-shelf capabilities.

We modeled a typical split-cylinder test setup using a $15.2 \mathrm{~cm}$ dia $\times 30.5 \mathrm{~cm}$ cylinder of plain concrete with $\mathrm{f}_{\mathrm{c}}$ of $27.6 \mathrm{MPa}$ and density of $2400 \mathrm{~kg} / \mathrm{m}^{3}$. Note that $\mathrm{f}_{\mathrm{c}}$. for the dynamic concrete material models for ALE3D and ABAQUS was significantly higher. The spacers $(2.54-\mathrm{x} 2.54-\mathrm{x} 30.5 \mathrm{~cm})$ were modeled with elastic properties simulating wood with elastic modulus (E) of $10342 \mathrm{MPa}$ and density of $530 \mathrm{~kg} / \mathrm{m}^{3}$. The upper spacer was given downward constant velocities of 20-, 100-, 500-, and $2500 \mathrm{~cm} / \mathrm{sec}$ (corresponding to average strain rates of $1.3,6.6,30.8,164.0 \mathrm{~s}^{-1}$, respectively) while the base spacer was held fixed. Figures 5 and 6 show typical concrete damage patterns. Force versus displacement of the upper spacer (P and y-direction in Figure 4) predicted by the software (Figures 7-10) and material damage were monitored during each solution. Simulation models for the five codes are summarized below:

ABAQUS - The ABAQUS mesh consisted of a full 3-D mesh, with approximately 190,000 nodes and three parts (the concrete cylinder and two wooden spacers). Contact was modeled using the Contact Inclusions, All Exterior option with penalty scaled to 0.1 and friction of 0.9 between surfaces. The concrete material model was Concrete Damaged Plasticity. Boundary conditions as described above were applied to the upper and lower spacers to achieve the desired loading.

LS-DYNA - The LS-DYNA mesh and boundary conditions were identical to the ABAQUS model using Contact_Automatic_General with the soft constraint option set to 1 , and a moving rigid planar surface in contact with the upper spacer. The concrete material model was Material 159, Continuous Surface Cap Model with damage and element erosion.

CTH - In CTH, we were not able to directly address the problem as defined above, as we were unable to produce a constant-velocity boundary condition to describe the upper platen motion. This is not to assert that such a capability does not exist, but if it does, it was not obvious to us. We tried two alternative solutions: an inflow boundary approximation, wherein the upper platen was represented by a block extending beyond the computational domain; and a very massive platen with a prescribed initial velocity. As neither approach proved satisfactory, we abandoned $\mathrm{CTH}$ for this particular problem.

ALEGRA - The Alegra simulation was modeled in 2-D $(1 \mathrm{~cm}$ long cylinder) using a plane strain Lagrangian formulation with approximately 16,000 elements. Because Alegra, like ALE3D, does not support contact in 2-D, the spacer meshes were merged with the concrete mesh. Alegra supplies the Brittle
Fracture Kinetics (BFK) combined material model for concrete, including submodels for the equation of state, yield, radial return, and fracture. This rather complex model employs up to 90 input parameters, making development of a user-defined concrete a daunting task. One pre-defined model, $S A C 5$, is available, representing a small-aggregate concrete with a native unconfined compressive strength near 6000 psi. This model was modified to achieve the desired strength and density with two parameters.

ALE3D- The ALE3D model consisted of a 3-D mesh $1 \mathrm{~cm}$ long, with approximately 25,000 nodes, two domains, two slide surfaces, and two regions (the concrete cylinder and two wooden spacers) using symmetry to limit the extent of the mesh in the axial direction. The concrete with porous crush model (Model 63) was used although other concrete models are available.

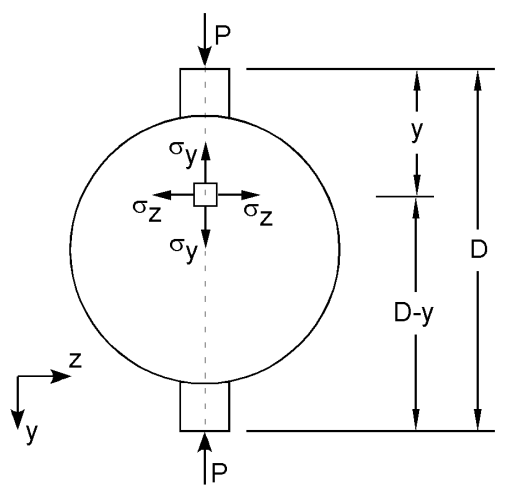

Figure 4. 2-Dimensional schematic of split-cylinder test

Lambert and Ross [15] have published fracture property experimental data from quasi-static and dynamic tests on 51 holed-notched concrete cylinder specimens. Normal stress information from quasi-static splitting tension tests conducted using a servo-tension device and from dynamic splitting tension tests conducted using a SHPB to achieve strain rates from 1 to $8 \mathrm{~s}^{-1}$ are given. Although the notched hole cylinders tested are physically different from the models discussed in this paper, it is insightful to compare these experimental results to the current computational results since actual split-cylinder tests at these strain rates are problematic. Lambert and Ross present their data in terms of normal stress as a function of strain rate (Figure 5 in [15]) with curve fits for each of their specimen groups. Note that the low strain rate values in [13] are reasonably consistent with the quasi-static values (Table 1 in [15]).

For the split-cylinder test, the load on the specimen is related to the stress normal to the load (splitting tensile stress) by

$$
\sigma_{Z}=\frac{2 P}{\pi L D} \text { or } P=\pi L R \sigma_{Z}=C \sigma_{Z}
$$


where $\sigma_{z}$ is the splitting tensile stress, $\mathrm{P}$ is the applied load (see Figure 4), $\mathrm{L}$ is the concrete cylinder length, D and $\mathrm{R}$ are the cylinder diameter and radius, respectively, and $\mathrm{C}$ is equal to 72.97 (using our nominal cylinder dimensions, stress in $\mathrm{MPa}$, and load in $\mathrm{kN}$ ). Using this equation, we converted the ranges of splitting tensile stresses from tests reported in [15] to maximum forces corresponding to our split-cylinder simulations. These data ranges were plotted as bands on Figures 7 and 8 for comparison with simulation results.

\section{Discussion}

ABAQUS - Several iterations of the simulation were attempted before a working model was achieved including modification of the scale penalty in the contact controls and using a friction coefficient between the contact surfaces. The initial concrete material model, Brittle Cracking, would only solve using an amplitude ramp function resulting in strain-rates significantly different from those used for the other codes.

The Concrete Damaged Plasticity (CDP) model was used for the results reported here. This material model seemed more robust and ran without the amplitude ramp. This material model has been used successfully at the INL for impact simulations giving results in agreement with published data [16] and indicating that the CDP model is insensitive to reasonable values of concrete strength $\left(\mathrm{f}^{\prime}{ }_{\mathrm{c}}\right)$. All of the ABAQUS models took several days to run even with multiple processors. Perhaps input modifications could result in shorter simulations times. The boundary conditions were easy to apply and the results were easy to extract using the ABAQUS postprocessor, Viewer. Results shown in Figures 7-10 are in relative agreement with those predicted by LS-DYNA.

LS-DYNA - Simulations were relatively straight forward using general contact options and a well documented concrete material model with strain rate effects. Care in preparing the mesh with adequate element density in the contact regions, small initial gaps between parts, and use of a contact softening option yielded a working input model without extensive user modifications. LS-DYNA has numerous built in features (i.e.: capturing the equivalent force on a rigid surface, automatic contact surface generation, and damaged element erosion) that greatly reduce the user effort in both pre- and post-processing. All LS-DYNA simulations ran in a few minutes to a few hours on eight processors. LS-DYNA is well suited to modeling impacts in this range of velocities and strain rates.

Results from LS-DYNA show maximum tensile force in relative agreement with representative test data. Strain rate effects are included in the concrete material model and can be turned off to simulate static results (see Figure 7).

CTH - We were unable to solve this problem with CTH with our current user knowledge base.

ALEGRA - Meshing of the model, and applying loads and boundary conditions was very nearly trivial for this problem. More attention was necessary to correctly implement the concrete material model, and record reaction at the prescribed-velocity boundary. To extract reaction forces, tracer particles had to be inserted along the node lines of interest, so that the reaction plot variable, which is tracer-based, could be recorded to the history database. Reaction values were then multiplied by the length of the cylinder to get values comparable to the 3-D results. Finally, in order to generate spreadsheet tables of reaction force vs. displacement, a translation script was written to translate the history database to spreadsheet format.

The Alegra results show a slightly higher peak force than reported by the other codes, as expected due to the plane-strain approach, which neglects the end effects of finite-length cylinders. Alegra's reported reaction force also appears to retain too much strength beyond the peak and at higher load rates exhibits significant oscillation. This behavior is surmised to be an artifact of the Lagrangian approach taken here which lacks a damage based element erosion capability. An obvious extension would be to run the problem in both Eulerian and ALE modes - precluded in the current effort by time and budget constraints.

ALE3D- The model was prepared using the internal ALE3D mesh generator and some basic input model setups included with the code distribution. Meshing of the concrete cylinder and the wooden platens was straightforward given some experience with the internal mesh generator. Likewise, implementation of the platen boundary conditions (velocity on the upper and no translation on the lower) was also straightforward. Displacement and reaction forces were captured using history variables and extensive variable manipulation logic available to add element reaction forces, scale the result by the cylinder length, and save the history data base in spread sheet format. Contact interaction between the platen and concrete cylinder meshes was accomplished through use of ALE3D slide surfaces. Slide surfaces require a 3-D model since slides must be at least two elements wide. Although several concrete models are available in ALE3D, only the porous crush with damage representation was used in the current simulations. Although the $\mathrm{f}^{\prime}{ }_{\mathrm{c}}$ in the concrete model used was somewhat higher than desired, adjusting the various input parameters was not undertaken due to the complexity of the model and associated crush tables.

ALE3D results at the lowest strain rate differ from test data and the other simulation results. At the higher strain rates, ALE3D results are in better agreement with the other codes. All the ALE3D simulations were robust and ran in a few minutes to a few hours save the $20 \mathrm{~cm} / \mathrm{s}$ case where Courant limits required more than 14 hours using 2 processors. Obvious extensions would be investigation of advection to limit mesh distortions noted late in the transients, implicit hydro options to mitigate the Courant limitations and speed run times, and in-depth exploration of the effect of the various parameters in the concrete model. 


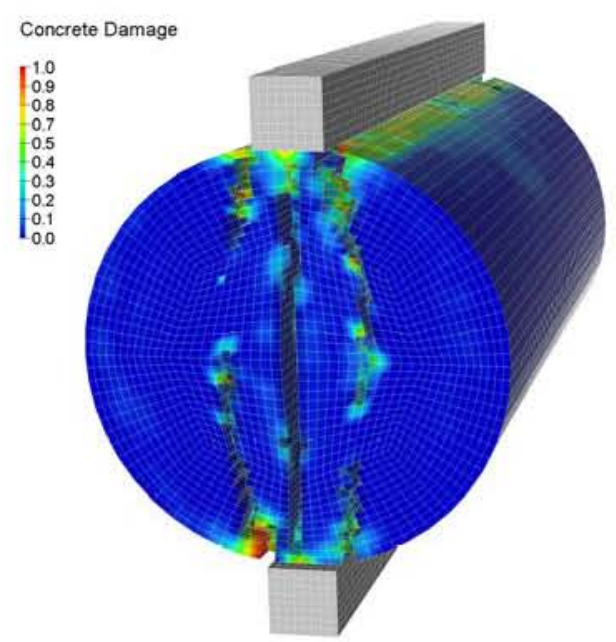

Figure 5. LS-DYNA model of split-cylinder showing damage at peak force, $20 \mathrm{~cm} / \mathrm{sec}$ velocity simulation

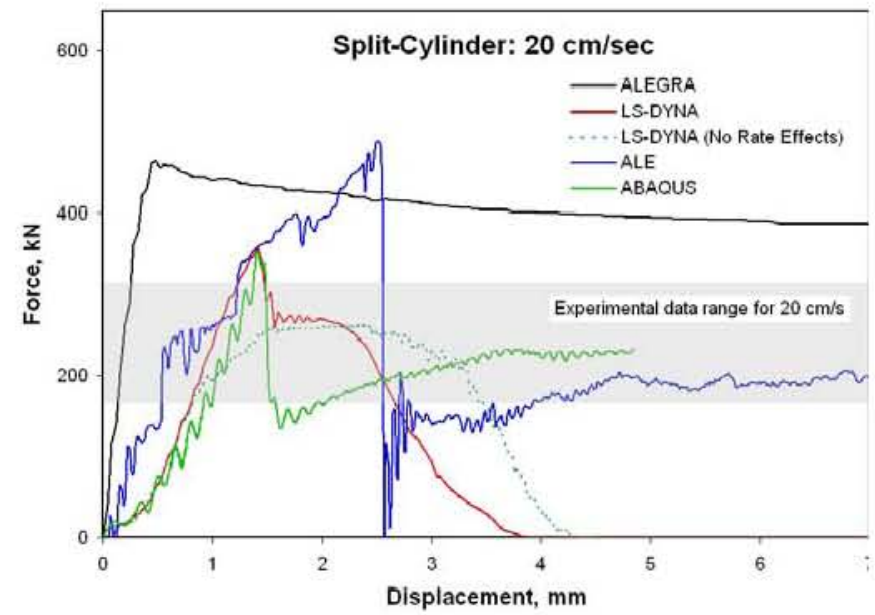

Figure 7. Split-Cylinder simulation results at $20 \mathrm{~cm} / \mathrm{sec}$, shaded area shows range of test data [15]

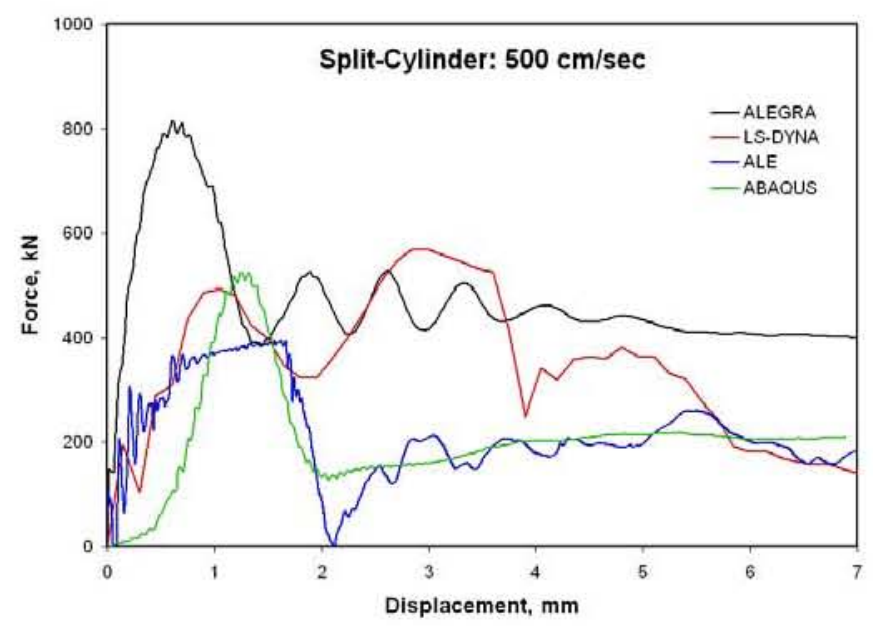

Figure 9. Split-Cylinder simulation results at $500 \mathrm{~cm} / \mathrm{sec}$

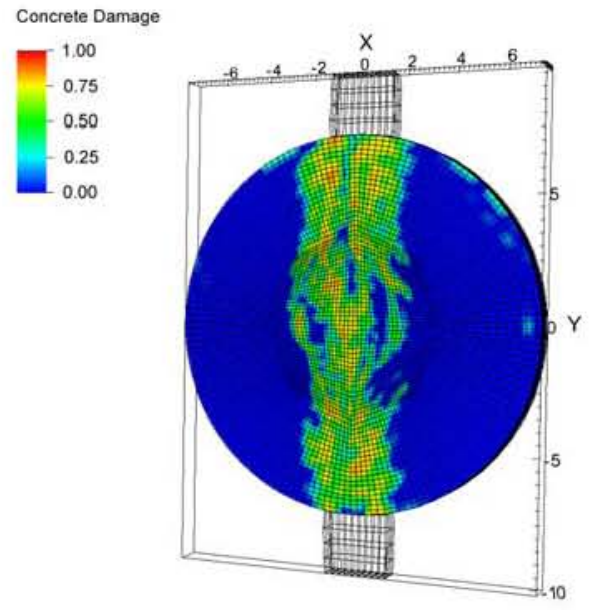

Figure 6. ALE3D model of split-cylinder showing damage at peak force, $20 \mathrm{~cm} / \mathrm{sec}$ velocity simulation

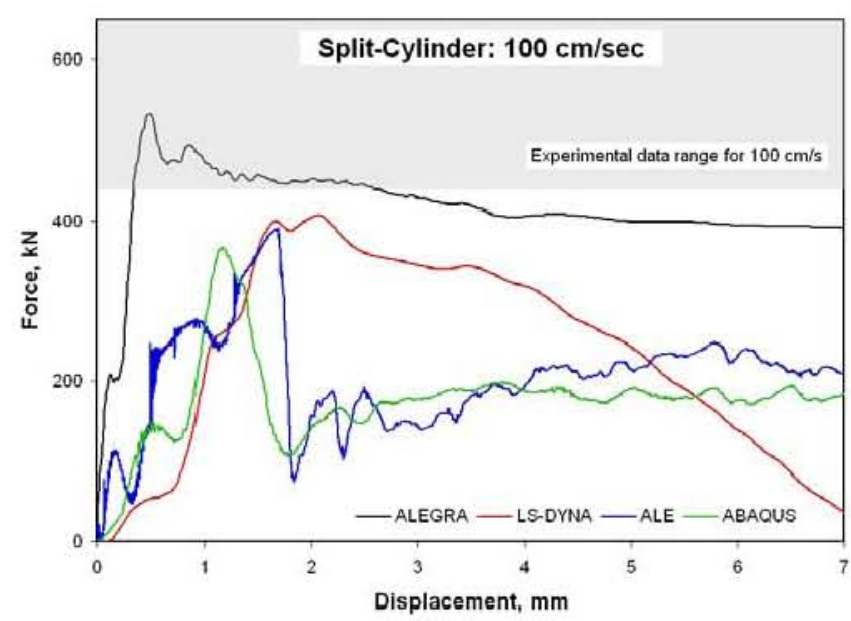

Figure 8. Split-Cylinder simulation results at $100 \mathrm{~cm} / \mathrm{sec}$, shaded area shows range of test data [15]

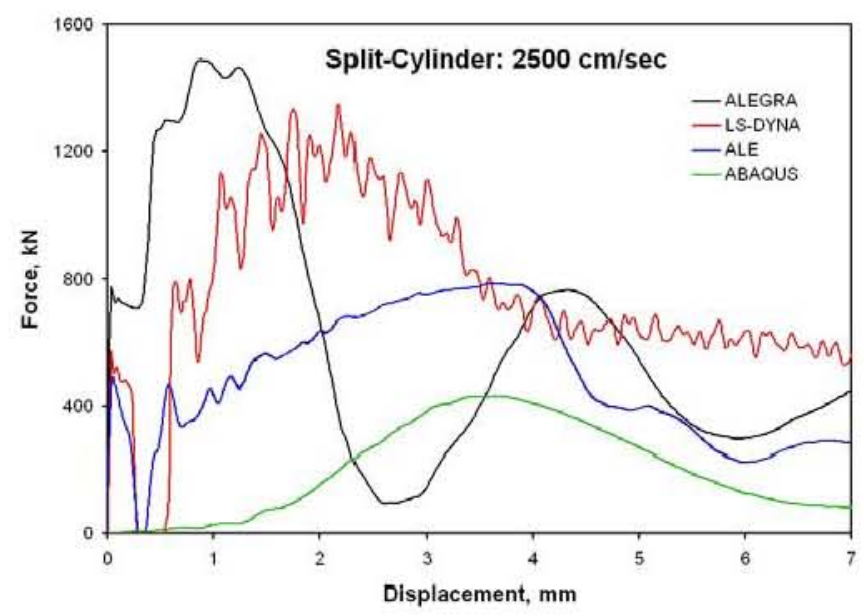

Figure 10. Split-Cylinder simulation results at $2500 \mathrm{~cm} / \mathrm{sec}$ 


\section{VENDOR SUPPLIED RESULTS}

The problem descriptions and sample input files for the Taylor Cylinder and Split-Cylinder benchmark problems were sent to two vendors at their request and solved using their inhouse analysts. Results for the Taylor Cylinder using both AUTODYN [7] and RADIOSS [8] (Figure 11) were virtually identical to those for the other codes using the Johnson-Cook [11] material model. Both used Lagrangian solvers and applied an initial velocity to all nodes.

The AUTODYN solution (Figure 12) for the concrete Split-Cylinder used an SPH solver and a concrete model developed by Klaus et. al. [17]. The RADIOSS solution (Figure 13) used Lagrangian techniques and material model R24, a plasticity based damage model developed specifically for concrete. Both codes used $\mathrm{f}_{\mathrm{c}}$, of $27.6 \mathrm{MPa}$ and density of $2400 \mathrm{~kg} / \mathrm{m}^{3}$. Results, especially the maximum force predictions, are comparable with results from the other codes shown in Figures 7-10.

Taylor Rod $19000 \mathrm{~cm} / \mathrm{s}$

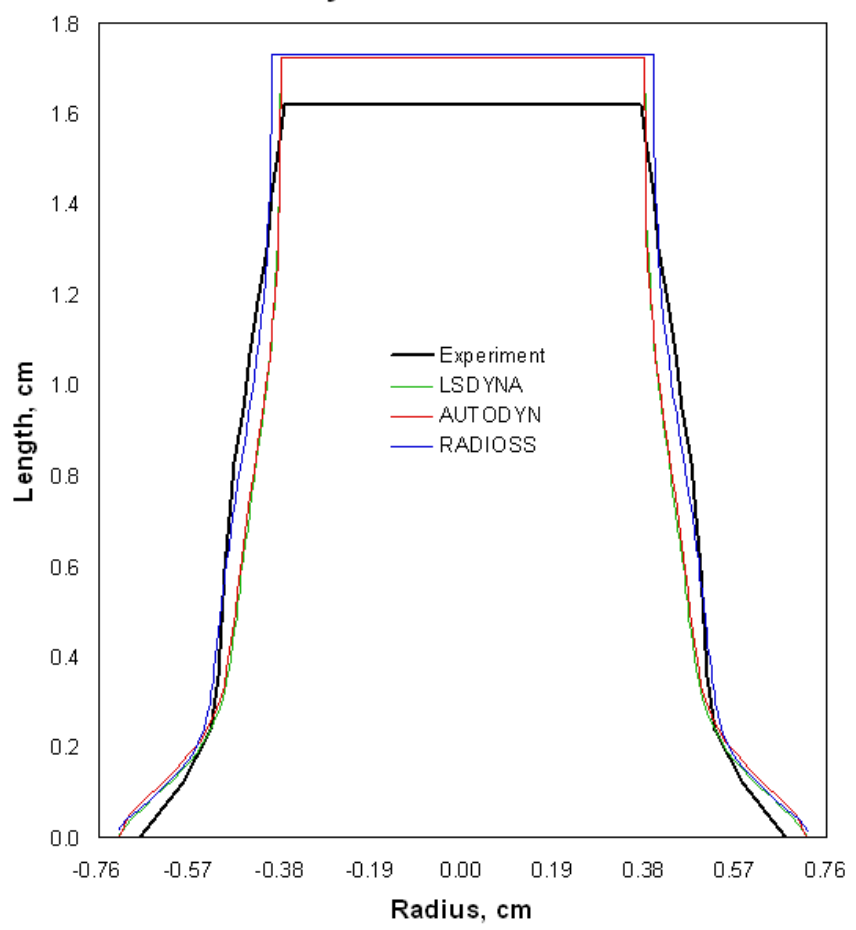

Figure 11. Comparison of AUTODYN and RADIOSS Taylor cylinder simulation results with LS-DYNA and experimental data, $19000 \mathrm{~cm} / \mathrm{sec}$

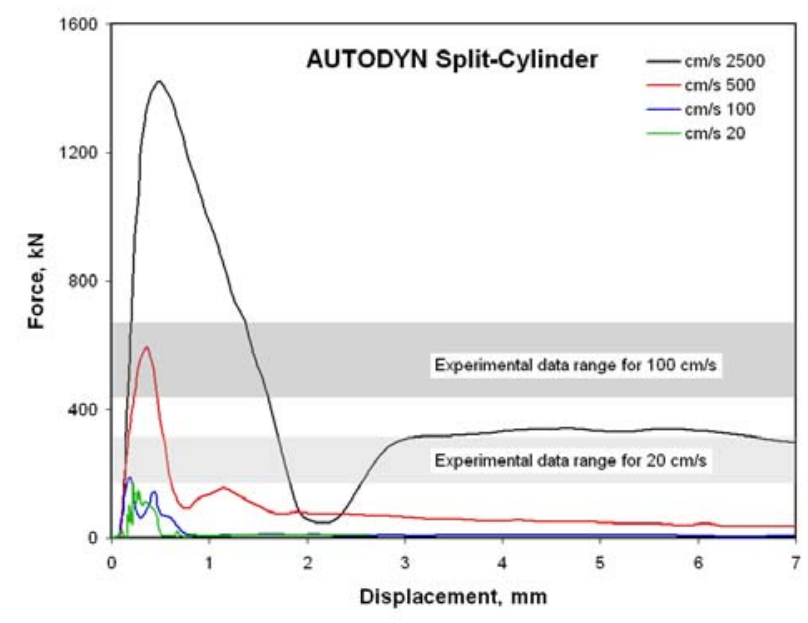

Figure 12. Split-Cylinder simulation results for AUTODYN, shaded area shows range of test data [15]

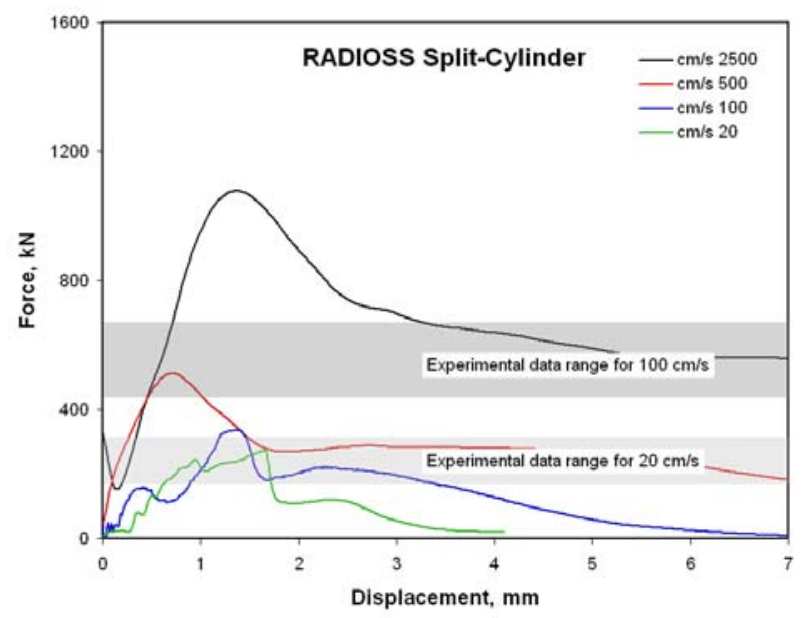

Figure 13. Split-Cylinder simulation results for RADIOSS, shaded area shows range of test data [15] 


\section{CONCLUSIONS}

Our investigation of the Taylor cylinder test with these five packages generated some points of interest:

All subject software agreed with one another very well, producing final deformed shapes nearly on top of one another, when using the same material model. For the material and velocity range herein, the different runs agreed very well over a broad range of mesh sizes (approximately a factor of three on element size) and dimensionalities, including 2-D axisymmetric, 2.5-D (solid with quarter symmetry), and 3-D.

As expected, the deformed cylinder shape was quite sensitive to the constitutive model employed, making it a valuable validation tool for new or suspect constitutive models and parameters.

The problem was quite straightforward to implement in all the analysis codes, making it an excellent introductory problem for new users of the codes, but less useful as a benchmark, as we could not discern significant differences among the codes with respect to ease of use, computational time, or accuracy. The Taylor cylinder problem can be considered a "go, no-go" problem that should be correctly solved by any software used to simulate the dynamic behavior of metals.

Regarding the dynamic split-cylinder tests, most simulation predictions were in relative agreement with respect to maximum force, but differ significantly in terms of energy as can be seen by comparing the areas under the forcedisplacement curves in Figures 7-10 and 12-13. It is interesting to note that use of element erosion (only used in LS-DYNA for this study) qualitatively predicted a cracking pattern.

There are three issues to consider with respect to comparing the peak load from the simulations to the published test data. First, the test data is quite broad in terms of peak load for a given displacement and strain-rate. Second, the models employed were quite diverse in terms of geometry and constitutive relationships. Third, the simulations did not model the test precisely. Future work should include more consistency among geometry and constitutive relationships and an attempt to model a specific test as accurately as possible. In terms of advancing simulations methods, the dynamic splitcylinder test invites the use of a multi-block mixed LagrangianEulerian approach, which may allow the desired prescribed velocity loading condition simultaneously with material splitting and separation typical of the Eulerian approach. This approach will be undertaken as part of a future effort.

\section{ACKNOWLEDGMENTS}

This work was sponsored by the U.S. Department of Energy under contract DE-AC07-05ID14517 and the INL Laboratory Directed Research and Development Program. The authors would like to thank Bence Gerber from ANSYS, Inc. and Amar Marpu, Julien Santini, and Ronald Thue from Altair Engineering, Inc. for providing the vendor solutions for the two benchmark problems.

\section{REFERENCES}

[1] Lacy, J. M., Novascone, S. R., Richins, W. D., Larson, T. K., "A Method for Selecting Software for Dynamic Event Analysis I: Problem Selection," Transactions 19th International Conference on Structural Mechanics in Reactor Technology, August 2007.

[2] ABAQUS, Getting Started with ABAQUS Version 6.6, ABAQUS, Inc. 2006.

[3] Hallquist, J. O., LS-DYNA Theory Manual, Livermore Software Technology Corporation, Livermore, CA, 2006.

[4] McGlaun, J. M., S. L. Thompson, and M. G. Elrick, $A$ Brief Description of the Three-Dimensional Shock Wave Physics Code CTH, Technical report SAND89-0607, Sandia National Laboratories, Albuquerque, NM, 1989.

[5] Carroll, S. K. et. al., ALEGRA:Version4.6 (Revised), Sandia National Laboratory report, SAND2005-draft, 2005.

[6] Nichols, Albert L. III (Ed.), Users Manual for ALE 3D, An Arbitrary Lagrange/Eulerian 3D Code System, Volume 1, Lawrence Livermore National Laboratory, UCRL-MA152204, Rev. 5, September 2006.

[7] ANSYS AUTODYN User Manual Version 11.0, ANSYS, Inc., Canonsburg, PA, 2008.

[8] RADIOSS Version 5.1, RADIOSS Theory Manual, Altair Engineering, Inc., Troy, MI, January 2008.

[9] Taylor, G. I., "The Use of Flat Ended Projectiles for Determining Yield Stress, Part I: Theoretical Considerations," Proceedings of the Royal Society (London), Vol. 194, 1948, p. 289-299.

[10] Johnson, G. R. and Holmquist, T. J., Test Data and Computational Strength and Fracture Model Constants for 23 Materials Subjected to Large Strains, High Strain Rates, and High Temperatures, LA-11463-MS (Limited Access), January 1989.

[11] Johnson, G. R., and Cook, W. H., "A Constitutive Model and Data for Metals Subjected to Large Strains, High Strain Rates and High Temperatures," Proceedings of the Seventh International Symposium on Ballistics (The Hague), ADPA, pp. 541-547, 1983.

[12] ASTM International, Standard Test Method for Splitting Tensile Strength of Cylindrical Concrete Specimens, C 496/C 496M-04, 2004.

[13]Hughes, M. L., Tedesco, J. W., and Ross, C. A., Numerical Analysis of High Strain Rate Splitting-Tensile Tests," Computers and Structures, Vol. 47, No. 4/5, pp. 653-671, 1993.

[14] Ruiz, G., Ortiz, M., and Pandolfi, A., "Three-Dimensional Finite-Element Simulation of the Dynamic Brazilian Tests on Concrete Cylinders," Int. J. Numer. Meth. Engng., Vol. 48, pp. 963-994, 2000. 
[15]Lambert, D. E., and Ross, C. A., "Strain Rate Effects on Dynamic Fracture and Strength," Int. J. Impact Eng. Vol. 24, pp. 985-998, 2000.

[16] Agardh, Magnusson, and Hansson, "High Strength Concrete Beams Subjected to Impact Loading, and Experimental Study," Defense Research Establishment, Sweden, December 1999.
[17] Klaus Thoma, Werner Riedel, Stefan Hiermaier, Mesomechanical Modelling of Concrete Shock Response, Experiments and Linking to Macromechanics by Numerical Analysis." ECCM'99, Munchen, Germany, 1999. 